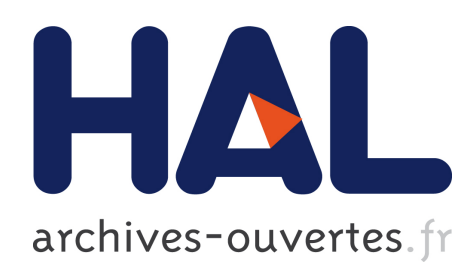

\title{
Sur la théorie du magnétisme
}

\author{
P. Langevin
}

\section{To cite this version:}

P. Langevin. Sur la théorie du magnétisme. 1905, 4 (1), pp.678-693. $<$ 10.1051/jphystap:019050040067800>. <jpa-00241050>

\section{HAL Id: jpa-00241050 \\ https://halv3-preprod.archives-ouvertes.fr/jpa-00241050}

Submitted on 1 Jan 1905

HAL is a multi-disciplinary open access archive for the deposit and dissemination of scientific research documents, whether they are published or not. The documents may come from teaching and research institutions in France or abroad, or from public or private research centers.
L'archive ouverte pluridisciplinaire HAL, est destinée au dépôt et à la diffusion de documents scientifiques de niveau recherche, publiés ou non, émanant des établissements d'enseignement et de recherche français ou étrangers, des laboratoires publics ou privés. 


\section{SUR LA THÉORIE DU MAGNÉTISME;}

Par M. P. IAANGEVIN.

I. Les propriétés magnétiques des corps, les manières diverses dont ils réagissent quand on les introduit dans un champ magnétique, se présentent tout d'abord comme extrêmement complexes: les substances ferromagnétiques prennent une aimantàtion intense, fonction non seulement du champ magnétisant actuel, mais de toute la série des champs antérieurs, grâce à la possibilité d'un magnétisme rémanent; d'autres corps s'aimantent faiblement dans le sens du champ magnétisant, proportionnellement à son intensité avec un coefficient, la susceptibilité paramagnétique, qui varie, comme l'a montré M. Curie, en raison inverse de la température absolue. De plus, l'expérience montre qu'une élévation de température fait passer une même substance de manière continue de l'état ferromagnétique à l'état paramagnétique, comme elle permet de passer de manière continue de l'état liquide à l'état gazeux.

Enfin d'autres corps, peut-être les plus nombreux, se polarisant très faiblement en sens inverse du champ magnétisant, sont diamagnétiques, sans qu'il soit possible d'observer aucune transition entre cette propriété et les deux premières, et, autre différence profonde, la susceptibilité diamagnétique se montre, sauf dans le cas du bismuth, rigoureusement indépendante de la température et de l'état physique.

On n'a donné jusqu'ici aucune représentation satisfaisante de cet ensemble complexe; l'hypothèse des courants moléculaires d'Ampère, reprise par M. Ewing, rend compte de certains faits de ferro et de paramagnétisme, mais sans indication sur l'origine de ces courants et sur la cause qui les maintient. De plus, Weber a supposé, pour représenter le diamagnétisme, l'existence dans les molécules de circuits de résistance nulle dans lesquels la création d'un champ extérieur provoque des courants induits tels que le flux total qui traverse les circuits reste nul ; le sens de ces courants, d'après la loi de Lenz, correspond au diamagnétisme. Maxwell, dans son Traité, a tenté d'unifier les hypothèses d'Ampère et de Weber sans obtenir de solution précise, faute d'indications sur la structure moléculaire.

II. Nous possédons aujourd'hui de telles indications, grâce à la 
théorie qui fait de la matière une agglomération d'électrons en mouvement, de centres électrisés des deux signes, dont on sait que leu r déplacement produit dans l'éther un champ magnétique analogue à celui d'un élément de courant, d'intensité proportionnelle à la charge de l'électron et à sa vitesse. La création de ce champ magnétique au moment de la mise en mouvement de l'électron implique une dépense d'énergie proportionnelle au carré de la vitesse, énergie de self-induction du courant équivalent, de même forme que l'énergie cinétique. Un centre électrisé possède donc une inertie par le fait seul qu'il est chargé, et il paraît vraisemblable aujourd'hui que toute l'inertie est ainsi d'origine électromagnétique, l'inertie d'une molécule matérielle n'étant que la somme des inerties électromagnétiques des électrons quil la constituent.

De plus, un rayonnement électromagnétique ou lumineux est émis chaque fois qu'un électron subit un changement de vitesse, une accélération, et il est naturel de chercher dans les électrons qui gravitent à l'intérieur des molécules, suivant des orbites fermées, l'origine des raies spectrales; la période des vibrations lumineuses, voisine de $10^{-13}$ secondes, doit être de l'ordre des durées $\tau$ de révolution des électrons sur leurs orbites.

On sait comment cette conception permit à M. Lorentz de prévoir certaines particularités du phénomène de Zeeman, que l'expérience confirma entièrement.

III. Il est naturel d'assimiler aux courants particulaires d'Ampère ces électrons gravitant sur des orbites fermées. On montre facilement qu'une semblable orbite, de surface $S$, parcourue pendant le temps $\tau$ par un électron de charge $e$, est équivalente, au point de vue de la production du champ magnétique moyen à l'extérieur, à un aimant normal au plan de l'orbite et de moment magnétique

$$
\mathrm{M}=\frac{e \mathrm{~S}}{\tau}=\mathrm{S} i
$$

autrement dit, l'orbite est équivalente à un circuit traversé par un courant d'intensité $\frac{e}{\tau}$.

Il n'est peut-être pas inutile de rappeler comment la production d'un champ magnétique par une particule électrisée, en mouvement par rapport à l'éther, est une conséquence nécessaire des équations de Hertz. Celles-ci, complètement vérifiées par l'expérience, tra- 
duisent en langage mathématique l'existence du courant de déplacement de Maxwell et des phénomènes d'induction, d'après lesquels, dans le vide, toute variation de l'un des deux champs électrique ou magnétique suffit pour créer l'autre :

$1^{\circ}$ L'intégrale du champ électrique le long d'un circuit fermé est égale à la dérivée par rapport au temps du flux de force magnétique qui traverse le circuit (induction);

$2^{\circ}$ L'intégrale du champ magnétique le long d'un circuit fermé est égale à la dérivée par rapport au temps du flux de force électrique qui traverse le circuil (courant de déplacement).

Ce dernier énoncé suppose que la surface limitée au circuit sur laquelle on calcule le flux n'est traversée par aucune charge électrique, par aucun électron.

Considérons maintenant une particule électrisée, $\mathrm{O}$, de charge $e$, se mouvant suivant $\mathrm{OX}$ avec la vitesse $v$ par rapport au milieu (supposé complètement immobile dans la théorie de Lorentz), et appliquons le second des énoncés précédents à une circonférence de rayon $\mathrm{AM}=l$ et de plan perpendiculaire à la direction $\mathrm{OX}$.

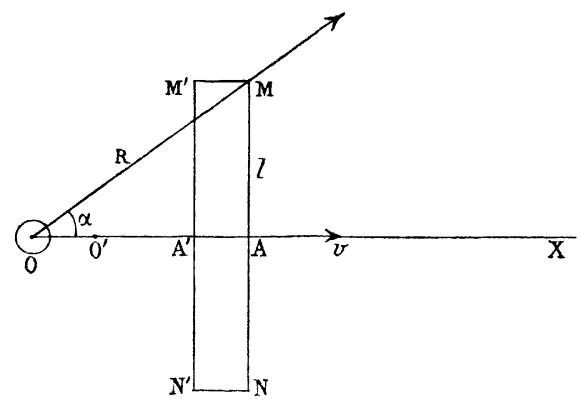

FIG. 1.

La particule électrisée étant supposée de révolution autour de OX, il est impossible que le champ magnétique, le même en tout point de la circonférence MN par raison de symétrie, soit nul sur cette circonférence, puisque le flux de force électrique qui la traverse varie par suite du mouvement de la particule. De plus, le champ magnétique $\mathrm{H}$ en $\mathrm{M}$ est nécessairement tangent à la circonférence, c'est-à-dire normal au plan de la figure, puisque tout le système est évidemment superposable à son image par rapport à ce plan, et qu'un 
champ magnétique, possédant la symétrie d'un cylindre tournant $\left({ }^{1}\right)$, n'a qu'un plan de symétrie normal à sa direction.

L'intégrale du champ magnétique le long de la circonférence de longueur $2 \pi l$ est :

$$
2 \pi l \mathrm{H} \text {. }
$$

Pour calculer la dérivée du flux de force électrique, cherchons la variation de ce flux quand la particule se déplace pendant le temps $d t$ de la quantité $\mathrm{OO}^{\prime}=v d t$. Le nouveau flux produit par $\mathrm{O}^{\prime}$ à travers $M N$ est égal à celui produit par $O$ à travers la circonférence $\mathrm{M}^{\prime} \mathrm{N}^{\prime}$ égale à $\mathrm{MN}$ et distante de celle-ci de $\mathrm{AA}^{\prime}=v d t$. Or la différence des flux produits par $O$ à travers les deux cercles est, par application du théorème de Gauss, égale au flux produit à travers la couronne cylindrique $\mathrm{MNM}^{\prime} \mathrm{N}^{\prime}$ qui forme avec les cercles une surface fermée ne contenant aucune charge à son intérieur. Ce flux est :

$$
\begin{aligned}
d \varphi & =\frac{e}{\mathrm{R}^{2}} \sin \alpha 2 \pi l v d t, \\
\frac{d \varphi}{d t}=2 \pi l \mathrm{H} & =\frac{e}{\mathrm{R}^{2}} \sin \alpha 2 \pi l v d t, \\
\mathrm{H} & =\frac{e v}{\mathrm{R}^{2}} \sin \alpha .
\end{aligned}
$$

C'est exactement le champ magnétique qui serait produit en $\mathbf{M}$ par un élément de courant de longueur òs placé en $\mathrm{O}$, parallèle à $\mathrm{OX}$, et de moment $i \grave{s}=e v$.

La valeur $\frac{e}{\mathrm{R}^{2}}$, prise pour le champ électrique en $\mathrm{M}$, suppose que ce champ n'est pas modifié par suite du mouvement, ce qui reste sensiblement exact tant que la vitesse $v$ ne devient pas voisine de celle de la lumière ; autrement, la loi d'induction montre que le champ magnétique produit réagit à son tour sur le champ électrique pour en changer la répartition, les lignes de force tendant, à distance de $O$ grande par rapport au rayon de la particule, à s'accumuler dans le plan perpendiculaire à $\mathrm{OX}$.

L'expression obtenue pour $\mathrm{H}$ reste utilisable même pour les électrons présents dans la molécule, puisque leur vitesse ne semble pas, en général, s'élever beaucoup au-dessus du centième de celle de la lumière.

(1) P. Cckik, J. de Pllys.. 3e série, t. 111, p. $\$ 13: 1894$. 
Il est facile, comme l'ont déjà fait divers auteurs, de calculer, en partant du résultat précédent, la projection $H_{z}$ sur une direction quelconque PM du champ magnétique $\mathrm{H}$ produit au point $\mathrm{M}$ par une particule électrisée $O$ décrivant une orbite fermée, de petites dimensions par rapport à la distance $R$, suivant une courbe dont la

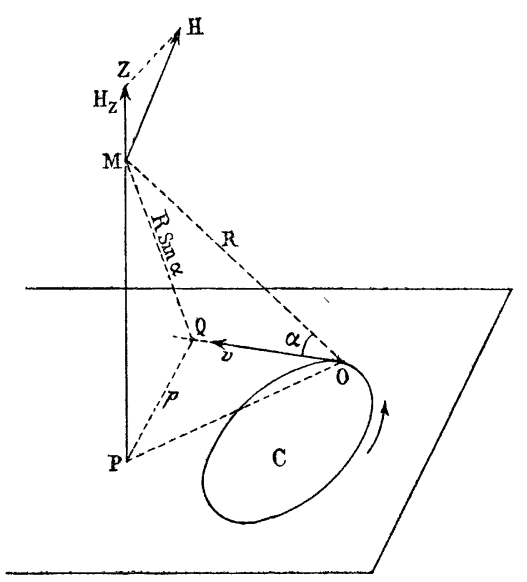

Fig. 2.

projection $\mathrm{C}$ sur un plan perpendiculaire à $\mathrm{PM}$ importe seule, puisque la composante de la vitesse parallèle à $\mathrm{PM}$ produit en $\mathrm{M}$ un champ magnétique perpendiculaire à $\mathrm{PM}$ et dont la projection est nulle. Soit $v$ la vitesse projetée. Si $p$ est la distance de cette vitesse au point $\mathrm{P}$, la similitude des triangles $\mathrm{PQM}$ et $\mathrm{ZMH}$ donne:

$$
\mathrm{H}_{z}=\mathrm{H} \frac{\mathrm{R}^{2}}{\mathrm{R} \sin \alpha},
$$

d'où, en remplaçant $H$ par sa valeur $\frac{\rho v \sin \alpha}{\mathrm{R}^{2}}$,

$$
\mathrm{H}_{z}=\frac{e p v}{\mathrm{R}^{3}} .
$$

Le moment $p v$ de la vitesse par rapport à PM est égal d'ailleurs au double de la vilesse aréolaire $\mathrm{A}$ du point $\mathrm{O}$ sur l'orbite projetée, par rapport au point $P$, et comptée positivement dans le sens trigonométrique.

Donc :

$$
\mathrm{H}_{z}=\frac{2 e \mathbf{A}}{\mathbf{R}^{3}} .
$$


Ce champ magnétique projeté $H_{z}$ restera constant dans le cas particulier où le mouvement de l'électron serait dû à des forces centrales émanant d'un point $\mathrm{C}$ intérieur à l'orbite, et où la direction MP passerait par ce point $\mathrm{C}$, puisque la vitesse aréolaire $\mathrm{A}$ resterait alors constante, ainsi que la distance $\mathrm{R}$ supposée grande par rapport aux dimensions de l'orbite.

Dans tous les autres cas, $\vec{H}_{z}$ sera variable, et la grandeur intéressante devient sa valeur moyenne $\mathrm{H}_{z}$ en fonction du temps. Si les variations de $\mathrm{R}$ quand $\mathrm{O}$ se déplace sont du second ordre par rapport aux dimensions de l'orbite, ce qui suppose $\mathbf{R}$ grand par rapport à $p$, c'est-à-dire $\alpha$ toujours voisin d'un angle droit, on peut considérer $\mathrm{R}$ comme invariable, et l'on a, si $\overline{\mathrm{A}}$ désigne la valeur moyenne de la vitesse aréolaire,

$$
\overline{\mathrm{H}}_{z}=\frac{2 e \overline{\mathrm{A}}}{\mathrm{R}^{3}} .
$$

Si S représente la surface de l'orbite projetée et $\tau$ la période de révolution, on a évidemment :

$$
\overline{\mathrm{A}}=\frac{\mathrm{S}}{\tau}
$$

donc

$$
\mathbf{H}_{z}=\frac{2 e}{\mathrm{R}^{3}} \frac{\mathrm{S}}{\tau} \text {. }
$$

Le courant particulaire est done équivalent, au point de vue de la production de ce champ moyen, à un aimant élémentaire de moment

$$
\mathrm{M}=\frac{e \mathrm{~S}}{\tau}=\overrightarrow{e \mathrm{~A}} \text {. }
$$

Il est facile de montrer que cette équivalence subsiste, pour les points éloignés de l'orbite, dans une direction quelconque, telle que les variations de $\mathrm{R}$ soient de l'ordre des dimensions de l'orbite. De sorte qu'au total le champ magnétique moyen produit par un courant particulaire à distance est identique à celui d'un aimant normal au plan de l'orbite et de moment $\frac{e \mathrm{~S}}{\tau}=e \overline{\mathrm{A}}$.

Une molécule contient autant de semblables courants particulaires qu'elle renferme d'électrons en mouvement. L'ensemble des aimants équivalents peut avoir un moment magnétique résultant nul : ce sera le cas si l'édifice moléculaire possède une symétrie assez élevée pour 
être incompatible avec la dissymétrie d'un moment magnétique, comparable à celle d'un cylindre tournant. Si la molécule a au contraire pour groupe de symétrie un intergroupe de celui du cylindre tournant, un moment résultant pourra exister et la molécule sera un petit aimant.

Nous allons voir que, dans tous les cas, la création d'un champ magnétique extérieur modifie les courants particulaires comme le supposait Weber, en polarisant diamagnétiquement toutes les molécules. Cet effet se produit seul si le moment résultant est nul : la matière est diamagnétique au sens ordinaire du mot. Si le moment résultant n'est pas nul, au phénomène diamagnétique initial se superpose un autre phénomène dû à l'orientation des aimants moléculaires par le champ extérieur : la substance est alors paramagnétique, si les actions mutuelles entre aimants moléculaires sont négligeables comme dans le cas des gaz ou des solutions, ferromagnétique dans le cas où ces actions mutuelles jouent le rôle essentiel. Dès que le paramagnétisme apparaît, il est énorme par rapport au diamagnétisme initial et le masque complètement. Ceci explique l'absence de continuité entre le para et le diamagnétisme : la substance possède ou ne possède pas la dissymétrie du cylindre tournant; le paramagnétisme existe ou non, et, quand il existe, il masque complètement le diamagnétisme initial sous-jacent.

IV. On peut, en effet, démontrer que la création d'un champ magnétique extérieur, qui, d'après les équations de Hertz-Maxwell, s'accompagne de la production d'un champ électrique, modifie le mouvement des électrons sur leurs orbites, de tous les électrons, dans le sens qui correspond au diamagnétisme, le moment magnétique d'un courant particulaire. quelconque supposé normal à la direction du champ $\mathrm{H}$ étant augmenté de

$$
\Delta \mathbf{M}=-\frac{\mathrm{H} e^{2} \mathrm{~S}}{4 \pi m},
$$

si $m$ est la masse de l'électron.

Je démontrerai ici cette formuleseulement dans le cas particulier d'un électron gravitant le long d'une orbite circulaire autour d'un centre qui l'attire suivant une loi quelconque en fonction de la distance. Le moment magnétique de l'aimant équivalent au courant particulaire est égal au produit de la charge $e$ de l'électron par la vitesse aréolaire (constante en l'absence de toute autre action que la force cen- 
trale) de l'électron par rapport au centre. Si $\omega$ est la vitesse angulaire de rotation et $r$ le rayon de l'orbite, on a :

$$
\mathrm{M}=e \mathrm{~A}=e \frac{\omega r^{2}}{2} \text {. }
$$

Si une force extérieure agit sur l'électron, telle que la force électrique induite par l'établissement du champ magnétique $\mathrm{H}$, il en résultera un changement de la vitesse aréolaire $A$, et, par suite, de $M$, en vertu de l'équation connue:

$$
2 m \frac{d \mathbf{A}}{d t}=\text { moment de la force extérieure. }
$$

Si $\mathrm{E}$ est la composante suivant la tangente à l'orbite du champ électrique induit, le moment de la force est Eer; donc :

$$
d \mathbf{M}=e d \mathbf{A}=\frac{e^{2}}{2 m} \mathrm{E} r d t .
$$

La vitesse de l'électron est $\omega r$, de sorte que l'élément $d s$ de trajectoire parcouru pendant le temps $d t$ est wrdt, d'où :

$$
d \mathbf{M}=\frac{e^{2}}{2 \omega m} \mathrm{E} d s
$$

et pour un tour, effectué pendant le temps périodique $\tau$ que nous supposerons, ce qui est toujours réalisé en pratique, très court par rapport à la durée d'établissement du champ magnétique, ò $\mathrm{M}$ étant la variation du moment pendant ce tour, durant lequel $\omega$ peut être considéré comme constant à cause de l'hypothèse précédente, on a :

$$
\delta \mathrm{M}=\frac{e^{2}}{2 \omega m} \int \mathrm{E} d s
$$

Or, d'après la loi d'induction rappelée plus haut, l'intégrale considérée, qui est l'intégrale curviligne du champ électrique prise le long de l'orbite, est égale à la dérivée du flux de force magnétique HS par rapport au temps :

$$
-\int \mathrm{E} d s=\frac{d \mathrm{HS}}{d t} \equiv \frac{1}{\tau} \delta \mathrm{HS},
$$

le signe - traduisant le sens indiqué par la loi de Lenz et en tenant compte de $\omega=\frac{2 \pi}{\tau}:$

$$
\delta \mathrm{M}=-\frac{e^{2}}{+\pi m} \delta \mathrm{HS} .
$$

J. de Phys., '́ érie, t. 1V. (Uctobre 190̈.) 
Pour la variation totale $\Delta \mathrm{M}$ qui résulte de l'établissement du champ $\mathrm{H}$, on en déduit :

$$
\Delta \mathrm{M}=-\frac{\mathrm{H} e^{2} \mathrm{~S}}{4 \pi m} .
$$

L'hypothèse faite sur la lenteur d'établissement du champ peut d'ailleurs être supprimée si l'on envisage, ce qui est nécessaire en pratique, l'effet moyen sur un grand nombre d'orbites analogues orientées de toutes les manières possibles.

Cette modification diamagnétique est d'ailleurs indépendante de la manière dont on a réalisé la superposition du courant particulaire et du champ extérieur, soit par création de celui-ci au moyen d'un courant, soit par déplacement de la molécule dans un champ préexistant. Elle est toujours extrêmement faible : on a en effẹt

$$
\frac{\Delta \mathrm{M}}{\mathrm{M}}=\frac{\mathrm{H} \tau}{4 \pi} \frac{e}{m}<10^{-9} \mathrm{H},
$$

puisque la plus grande valeur connue de $\frac{e}{m}$ est $1,8 \times 10^{7}$ qui correspond aux corpuscules cathodiques, et que $\tau$ est de l'ordre $10^{-15}$. Les champs les plus intenses que nous sachions produire, toujours inférieurs $\dot{a} 10^{3}$, ne modifient donc pas de plus du dix-millième le moment magnélique des courants particulaires.

V. La petitesse de cette modification est justifiée par la petitesse du phénomène de Zeeman connexe de la modification diamagnétique. En effet, la variation d'intensité des courants particulaires produite au moment de l'établissement du champ correspond à un accroissement de période des électrons qui gravitent dans un sens, une diminution pour ceux qui gravitent en sens inverse, conformément aux changements qui constituent l'effet Zeeman. L'extrême petitesse de celui-ci justifie ce qui précède sur la petitesse de la modification diamagnétique.

De plus, la généralité du phénomène de Zeeman prouve la généralité de l'effet diamagnétique. Même dans le fer, qui manifeste l'effet Zeeman, les courants particulaires sont modifiés dans le sens diamagnétique.

Il est d’ailleurs intéressant de móntrer que, dans le cas particulier supposé plus haut d'une orbite circulaire avec une force centrale quelconque $f(r)$, l'établissement du champ magnétique ne modifie pas le rayon de l'orbite, mais seulement la durée de révolution de 
l'électron, du moins tant que le champ magnétique est assez faible, ce qui est toujours réalisé, comme on vient de le voir, pour ne produire qu'une très petite modification. En l'absence du champ magnétique, la condition du mouvement stationnaire est :

$$
f(r)=m \omega^{2} r .
$$

Supposons qu'en présence du champ magnétique $\omega$ et $r$ soient tous deux modifiés, de $\Delta \omega$ et $\Delta r$ respectivement; la nouvelle condition de mouvement stationnaire s'écrira, en tenant compte de la force magnétique Hewr:

$$
f(r+\Delta r)=m(\omega+\Delta \omega)^{2}(r+\Delta r)+\mathrm{H} e \omega r,
$$

et, en conservant seulement les termes du premier ordre :

$$
\left[f^{\prime}(r)-m \omega^{2}\right] \Delta r=2 m \omega r \Delta \omega+\mathrm{H} e \omega r .
$$

D'autre part, la grandeur obtenue plus haut pour la modification diamagnétique donne :

$$
\Delta \mathrm{A}=\Delta \frac{\omega r^{2}}{2}=-\frac{\mathrm{H} e}{4 m} r^{2}-4 m \omega \Delta^{2} r=2 m \omega r \Delta \omega+\mathrm{H} e \omega r .
$$

En retranchant de (2), il vient :

$$
\left[f^{\prime}(r)+3 m \omega^{2}\right] \Delta r=0 .
$$

Ce qui conduit à la condition :

$$
\Delta r=0, \quad \Delta \omega=-\frac{\mathrm{H} e}{2 m},
$$

à moins que la loi d'action ne satisfasse à la relation toute particulière :

$$
f^{\prime}(r)+3 m \omega^{2}=0
$$

qui, jointe à (1), donne :

$$
\begin{aligned}
& \frac{f^{\prime}(r)}{f(r)}=-\frac{3}{r}, \\
& f(r)=\frac{\mathrm{K}}{r^{3}} .
\end{aligned}
$$

Donc, à moins que la force attractive qui maintient l'électron sur l'orbite ne varie exactement en raison inverse du cube de la distance, la modification diamagnétique ne changera pas les dimensions de l'orbite, mais seulement la durée de révolution, de la manière qui oorrespond au phénomène de Zeeman. 
VI. Les courants particulaires se comportent comme le feraient des circuits de résistance nulle et de self-induction convenable. En effet, un semblable circuit, de self-induction L, serait modifié par le champ extérieur de manière que le flux total reste constant:

$$
\frac{d \mathrm{~L} i}{d t}=-\frac{d(\mathrm{HS})}{d t}
$$

d'où pour l'établissement du champ $\mathrm{H}$ :

$$
\Delta \mathrm{L} i=-\mathrm{HS} .
$$

Et le circuit considéré sera assimilable au courant particulaire si

$$
\begin{aligned}
\Delta \mathrm{M} & =-\frac{e^{2}}{4 \pi m} \mathrm{HS}=\frac{e^{2}}{4 m \pi} \Delta \mathrm{L} i, \\
\mathrm{~L} & =\frac{4 \pi m}{e^{2}} \frac{\mathrm{M}}{i}=\frac{4 \pi^{2} r^{2} m}{e^{2}},
\end{aligned}
$$

si l'orbite est une circonférence de rayon $r$.

L'énergie magnétique du circuit sera :

$$
\frac{\mathrm{L} i^{2}}{2}=\frac{4 \pi^{2} r^{2} m}{2 \tau^{2}}=m \frac{v^{2}}{2}
$$

c'est-ḋ-dire que le courant particulaire sera complètement assimilable $\dot{a}$ un circuit de résistance nulle, si l'inertie de l'électron qui le constitue est tout entière électromagnétique, si l'énergie cinétique $\frac{m v^{2}}{2}$ de l'électron est tout entière sous forme magnétique.

La modification diamagnétique étant extrêmement faible, les courants particulaires pourront être considérés comme indéformables et d'intensité sensiblement constante, et l'on obtiendra une représentation complete des faits du paramagnétisme et des échanges d'énergie entre aimants el courants dans l'hypothèse où les aimants sont constitués de semblables circuits auxquels les lois de l'électrodynamique ordinaire sont rigoureusement applicables.

VII. Si les molécules ont un moment résultant nul, aucune action du champ extérieur ne suivra la modification diamagnétique, aucun changement de leur mouvement d'ensemble. Les chocs ultérieurs entre molécules se produiront comme auparavant, aucun changement de température ne se montrera, et la propriété diamagnétique acquise subsistera seule. 
Inversement un changement de température ne modifiera pas cette propriété : tous les électrons intérieurs à la molécule contribuent à la produire, et le mouvement de ceux-ci, comme le montre l'invariabilité des raies spectrales, ne dépend pas de la température. On obtient ainsi la loi trouvée expérimentalement par M. Curie sur l'invariabilité de la constante diamagnétique avec la température.

Si l'on cherche, en partant des constantes diamagnétiques expérimentales, à calculer ce que doivent être les surfaces des orbites telles que $\mathrm{S}$, on trouve un résultat tout à fait conforme à ce que pouvaient faire prévoir la connaissance des dimensions moléculaires et l'hypothèse que les orbites sont intra-moléculaires. La petitesse des effets diamagnétiques est nécessitée par la petitesse des dimensions moléculaires.

VIII. Si les molécules ont un moment résultant non nul, elles subissent tout d'abord la modification diamagnétique tout à fait générale, puis le champ extérieur tend à faire tourner les axes magnétiques pour les rendre parallèles à sa direction; il en résulte un changement du mouvement d'ensemble des molécules, de leur agitation thermique, qui correspond à une inégale répartition de l'énergie cinétique entre les molécules, celles dont l'axe magnétique est parallèle au champ ayant une énergie cinétique plus grande que celle des autres. Ceci est incompatible avec l'équilibre thermique et, par l'intermédiaire des chocs mutuels, s'il s'agit d'un gaz, un réarrangement se produira après lequel la température sera uniforme et les aimants moléculaires dirigés de préférence dans le sens du champ extérieur, après lequel la propriété paramagnétique aura apparu. S'il résulte de ce réarrangement un accroissement $d \mathrm{M} d u$ moment magnétique résultant, le système producteur du champ magnétique fournit au mouvement d'ensemble des molécules, un travail qui, dans le cas dun corps gazeux ou dissous, se traduit par une élévation de température du milieu et est donné par:

$$
d \mathrm{~W}=\mathrm{H} d \mathrm{M}
$$

et, pour maintenir le gaz à température constante, il faut lui enlever une quantité de chaleur équivalente,

$$
d \mathbf{Q}=\mathbf{H} d \mathbf{M}=\mathbf{H}\left(\frac{\partial \mathbf{M}}{\partial \mathrm{H}} d \mathrm{H}+\frac{\partial \mathbf{M}}{\partial \mathrm{T}} d \mathbf{T}\right)
$$


en écrivant que $\frac{d Q}{\mathrm{~T}}$ est une différentielle exacte, on obtient la relation:

$$
\mathrm{M}=f\left(\frac{\mathrm{H}}{\mathrm{T}}\right)
$$

et, dans le cas d'une aimantation proportionnelle au champ,

$$
\mathrm{M}=k \frac{\mathrm{H}}{\mathrm{T}} \text {; }
$$

c'est la loi de M. Curie sur la susceptibilité paramagnétique inversement proportionnelle à la température absolue.

La comparaison suivante fera mieux comprendre la théorie qui précède : imaginons une masse gazeuse contenue dans un récipient fermé et soustraite à l'action de la pesanteur ; les molécules se répartiront de manière que la densité du gaz soit la mème en tous points, de même qu'en l'absence d'un champ magnétique extérieur les molécules d'un gaz magnétique tel que l'oxygène ont leurs axes répartis uniformément dans toutes les directions.

Si l'on vient à créer le champ de gravitation, les molécules prendront une accélération dirigée vers le bas, et, en l'absence de chocs mutuels, chaque molécule aura une vitesse plus grande en bas qu'en haut du récipient; mais cette inégalité de vitesses est incompatible avec l'équilibre thermique, et un réarrangement aura lieu grâce aux chocs mutuels, à la suite duquel s'établit la répartition donnée par la formule du nivellement barométrique : le centre de gravité s'est abaissé et, pour maintenir le gaz à la température initiale, il faut lui enlever une quantité de chaleur équivalente au produit du poids du gaz par cet abaissement du centre de gravité. On déduirait d'un raisonnement thermodynamique analogue à celui qui précède que cet abaissement du centre de gravité est inversement proportionnel à la température absolue.

Le raisonnement thermodynamique ne permet pas de calculer la fonction $f(T)$ dans l'expression (3) du moment magnétique. Pour aller plus loin, il est nécessaire de faire intervenir des considérations cinétiques et de déterminer, quand l'équilibre thermique est rétabli, la répartition des molécules aimantées entre les diverses directions, ou, dans le cas de la pesanteur, la variation de densité avec la hauteur donnée par la formule du nivellement barométrique.

Après le réarrangement, dans une masse de gaz à température 
uniforme, la répartition des molécules se fait entre les diverses régions de manière que les molécules soient plus nombreuses là où l'énergie potentielle est la plus faible, c'est-à-dire aux points les plus bas dans le cas de la pesanteur. La loi suivant laquelle se fait cette répartition est la généralisation donnée par M. Boltzmann( ${ }^{1}$ ) de la loi du nivellement barométrique. Le rapport des densités du gaz en deux points entre lesquels l'énergie potentielle d'une molécule varie de W est :

$$
e^{\frac{\mathrm{w}}{r \mathrm{~T}}}
$$

$e$ est la base des logarithmes népériens, T la température absolue du gaz et $r$ la constante de l'équation des gaz parfaits rapportée à une molécule, constante telle, d'après la théorie cinétique, que $r$ T représente les $\frac{2}{3}$ de l'énergie cinétique moyenne de translation, c'est-à-dire le double de l'énergie cinétique correspondant à chacun des trois degrés de liberté de cette translation.

Or on sait qu'une molécule comme celle de l'oxygène possède en outre deux degrés de liberté pour la rotation, comme l'indique la valeur de $\frac{\mathrm{C}}{c}$, à chacun de ces degrés de liberté correspondant une énergie cinétique moyenne égale aussi à $\frac{r \mathrm{~T}}{2}$, donc égale à $r \mathrm{~T}$ pour toute la rotation.

La répartition des molécules entre les diverses orientations sera déterminée par l'équilibre statique qui s'établira sous l'influence superposée de l'énergie potentielle magnétique - MH $\cos \alpha$ et de l'énergie $r$ T d'agitation thermique, les molécules étant de préférence orientées dans les directions de moindre énergie potentielle, c'està-dire avec leur axe magnétique vers la direction du champ. Si l'on répartit les axes magnétiques entre les diverses directions, la densité par unité d'angle solide variera d'une direction à l'autre proportionnellement à :

$$
e^{\frac{\mathrm{MH} \cos \alpha}{r \mathrm{~T}}},
$$

toutes les directions étant également probables si $\mathrm{M}$ ou $\mathrm{H}$ sont nuls.

(1) L. Boltzmann, Théorie des gaz, $1^{\text {re }}$ partie. Trad. Gallutti, p. $12 \pi$. 
Le nombre des molécules dont l'axe magnétique est dirigé dans l'angle solide $d \omega$ sera par suite:

$$
d n=\mathrm{K}^{\frac{\mathrm{MH}}{r \mathrm{~T}} \cos \alpha} d \omega .
$$

Prenons pour élément $d \omega$ une zone d'ouverture $d x$ autour de la direction du champ :

$\propto$ variant de 0 à $\pi$.

$$
d \omega=2 \pi \sin \alpha d \alpha
$$

Si $\mathrm{N}$ est le nombre total des molécules, $\mathrm{K}$ est déterminé; on doit avoir en effet, en posant :

$$
\begin{gathered}
\frac{\mathrm{MH}}{r \mathrm{~T}}=a, \\
\mathrm{~N}=2 \pi \mathrm{K} \int_{0}^{\pi} e^{a \cos \alpha} \sin \alpha d \alpha=2 \pi \mathrm{K} \int_{-1}^{+1} e^{a x} d x, \\
\mathrm{~N}=\frac{2 \pi \mathrm{K}}{a}\left(e^{a}-e^{-a}\right)
\end{gathered}
$$

ou

$$
\mathrm{K}=\frac{\mathrm{N} a}{4 \pi \mathrm{S} h a} .
$$

Le moment magnétique total des $\mathrm{N}$ molécules est évidemment dirigé parallèlement au champ et est égal à la somme des projections sur cette direction des moments composants. Pour l'unité de volume supposée contenir $\mathrm{N}$ molécules, ce moment résultant représente l'intensité d'aimantation I :

$$
\mathrm{I}=\int \mathrm{M} \cos \alpha d n=\int_{-1}^{+1} 2 \pi \mathrm{MK} x e^{a x} d x
$$

Or

$$
\int_{-1}^{+1} x e^{a x} d x=2\left(\frac{\mathrm{C} h a}{a}-\frac{\mathrm{S} h a}{a^{2}}\right)
$$

d'où

$$
\mathrm{I}=\mathrm{MN}\left(\frac{\mathrm{C} h a}{\mathrm{~S} h a}-\frac{1}{a}\right)
$$

Pour un nombre donné de molécules, une masse donnée du gaz, I, est donc bien fonction seulement de $a$, c'est-à-dire de $\frac{H}{\Gamma}$, conformément au résultat fourni par la thermodynamique.

IX. Si les actions mutuelles entre aimants moléculaires sont pos- 
HALDIÉ. - PHOTOMÉTRIE DANS LES SYSTÈmES OPTIQUES 693

sibles, si la substance est ferromagnétique, la chaleur dégagée n'est équivalente au travail fourni que pour un cycle fermé, et l'on obtient alors la chaleur d'hystérésis :

$$
Q=\int \mathrm{H} d \mathrm{M}
$$

Ce cas des corps ferromagnétiques se présente naturellement comme beaucoup plus complexe que les cas précédents du dia et du paramagnétisme, à cause de l'intervention des actions mutuelles entre aimants moléculaires, comme la théorie des milieux liquides et solides où interviennent les forces de cohésion est infiniment plus difficile et moins avancée que celle des gaz où la cohésion n'intervient pas de manière sensible. Les idées que je présente ici sont donc en quelque sorte l'équivalent magnétique de la théorie des gaz. Pour aller plus loin, il faudra étudier les actions directrices entre particules aimantées, et peut-être le cas le plus simple est-il celui des milieux cristallisés, sur les propriétés magnétiques desquels les travaux de M. Weiss nous ont donné déjà et nous promettent encore beaucoup d'indications précieuses, au même titre que les résultats de M. Maurain sur les dépôts électrolytiques de fer, où les actions directrices paraissent s'exercer à des distances considérables. 